\title{
Multimodal characterisation on FIB instruments combining nano-scale SIMS and SE imaging
}

Tom Wirtz ${ }^{1}$, Olivier De Castro ${ }^{2}$, Hung Quang Hoang ${ }^{2}$, Luca Cressa ${ }^{2}$, Santhana Eswara ${ }^{3}$, Antje Biesemeier $^{4}$ and Jean-Nicolas Audinot ${ }^{2}$

${ }^{1}$ Luxembourg Institute of Science and Technology (LIST), Belvaux, Luxembourg, ${ }^{2}$ Luxembourg Institute of Science and Technology (LIST), Luxembourg, ${ }^{3}$ Luxembourg Institute of Science and Technology, Belvaux, Luxembourg, ${ }^{4}$ Luxembourg Institute of Science and Technology (LIST), Belvaux, Not Applicable, Luxembourg

Critical questions in a wide range of scientific and technological fields can often only be solved by developing innovative characterisation tools. In this context, the structural characterisation and the chemical analysis at the nanometer scale are of particular relevance in a large variety of fields, ranging from the high-resolution imaging of dopant distributions in complex electronic devices to the generation of chemical maps of subcellular structures in biological samples to understand the underlying physiological processes. The following key characteristics are required and enabled in our instrument developments: (1) highest spatial resolution, (2) excellent chemical sensitivity, (3) high dynamic range (for the detection and mapping of elemental concentrations varying over several orders of magnitude) and (4) isotopic selectivity.

Secondary Ion Mass Spectrometry (SIMS) is an extremely powerful technique for analyzing surfaces, owing in particular to its ability to detect all elements from $\mathrm{H}$ to $\mathrm{U}$ and to differentiate between isotopes, its excellent sensitivity and its high dynamic range. SIMS analyses can be performed in different analysis modes: acquisition of mass spectra, depth profiling, 2D and 3D imaging. Adding SIMS capability to FIB instruments offers a number of interesting possibilities [1-2], including highly sensitive analytics, in-situ process control during patterning and milling, highest resolution SIMS imaging ( 10 $\mathrm{nm})$, and direct correlation of SIMS data with data obtained with other analytical or imaging techniques on the same instrument, such as high resolution secondary electron (SE) images or Energy-Dispersive X-Ray Spectroscopy (EDX) spectra.

In this global context, we developed several multi-modal FIB-based characterisation platforms using SIMS:

- DualBeam-SIMS, consisting of a Thermo Fisher DualBeam platform with a LIST magnetic sector SIMS system, allowing for the correlation of SE, EDX and SIMS data (Figure 1).

- HIM-SIMS, consisting of a ZEISS ORION NanoFab Helium Ion Microscope (HIM) with a LIST magnetic sector SIMS system, allowing for the correlation of SE and SIMS data (Figure 2) [3,4].

- npSCOPE, which is based on the Gas Field Ion Source (GFIS), providing finely focussed $\mathrm{He}^{+}$and $\mathrm{Ne}^{+}$ion beams, and further equipped with a SE detector, a LIST magnetic sector SIMS system and a novel position sensitive detector based on a microchannel plate with a delay line readout structure placed below the sample providing Scanning Transmission Helium Ion Microscopy (STHIM) data [5]. The npSCOPE hence allows for the correlation of in-situ SE, SIMS, bright field and dark field imaging [6].

- TEM-SIMS, consisting of an FEI Tecnai F20 Transmission Electron Microscope equipped with a $\mathrm{Ga}^{+}$FIB column and a dedicated SIMS system, allowing for the correlation of Transmission Electron Microscopy (TEM), EDX and SIMS data [7]. 
- SIMS:ZERO, which is a novel highest-resolution highest-sensitivity FIB-SIMS platform combining the latest generations of zeroK's high brightness $\mathrm{Cs}^{+}$ion source (based on the LowTemperature Ion Source (LoTIS) technology) and LIST's high-performance magnetic sector SIMS system with continuous focal plane detector. The use of $\mathrm{Cs}^{+}$as a primary ion enhances the ionisation of sputtered particles and hence further improves the analytical sensitivity.

To further enhance the potential of LIST's magnetic sector SIMS system, we have developed a novel continous focal plane detector based on a microchannel plate array covering the full focal plane of the magnetic sector and coupled to a readout structure based on capacitively coupled delay lines. With this new detector, the SIMS system allows for the detection of all masses in parallel for each single pixel, resulting in acquisition times as low as $1 \mathrm{~s}$ to obtain a full mass spectrum or 2 min to obtain a $512 \times 512$ pixel SIMS image with highest count rate and excellent dynamic range.

We have demonstrated that these FIB-SIMS instruments are capable of producing (i) a full mass spectrum with mass resolution $\mathrm{M} / \Delta \mathrm{M}=400-500$ for each single pixel, (ii) very local depth profiles making it possible to follow the chemical composition in real time during nano-patterning in FIB for applications such as end-pointing and (iii) elemental SIMS maps with a lateral resolution down to $12 \mathrm{~nm}$ [3-5]. Furthermore, FIB-SIMS opens the way for in-situ correlative imaging combining high resolution SE images, EDX data, TEM data, etc. with elemental and isotopic ratio maps from SIMS [2-4, 7].

Here, we will review the performance of the different instruments with a focus on new developments, showcase methodologies for high-resolution 3D chemical imaging, present a number of examples from various fields of applications (nanoparticles, battery materials, photovoltaics, micro-electronics, tissue and sub-cellular imaging in biology, geology,...) and give an outlook on new trends and prospects.
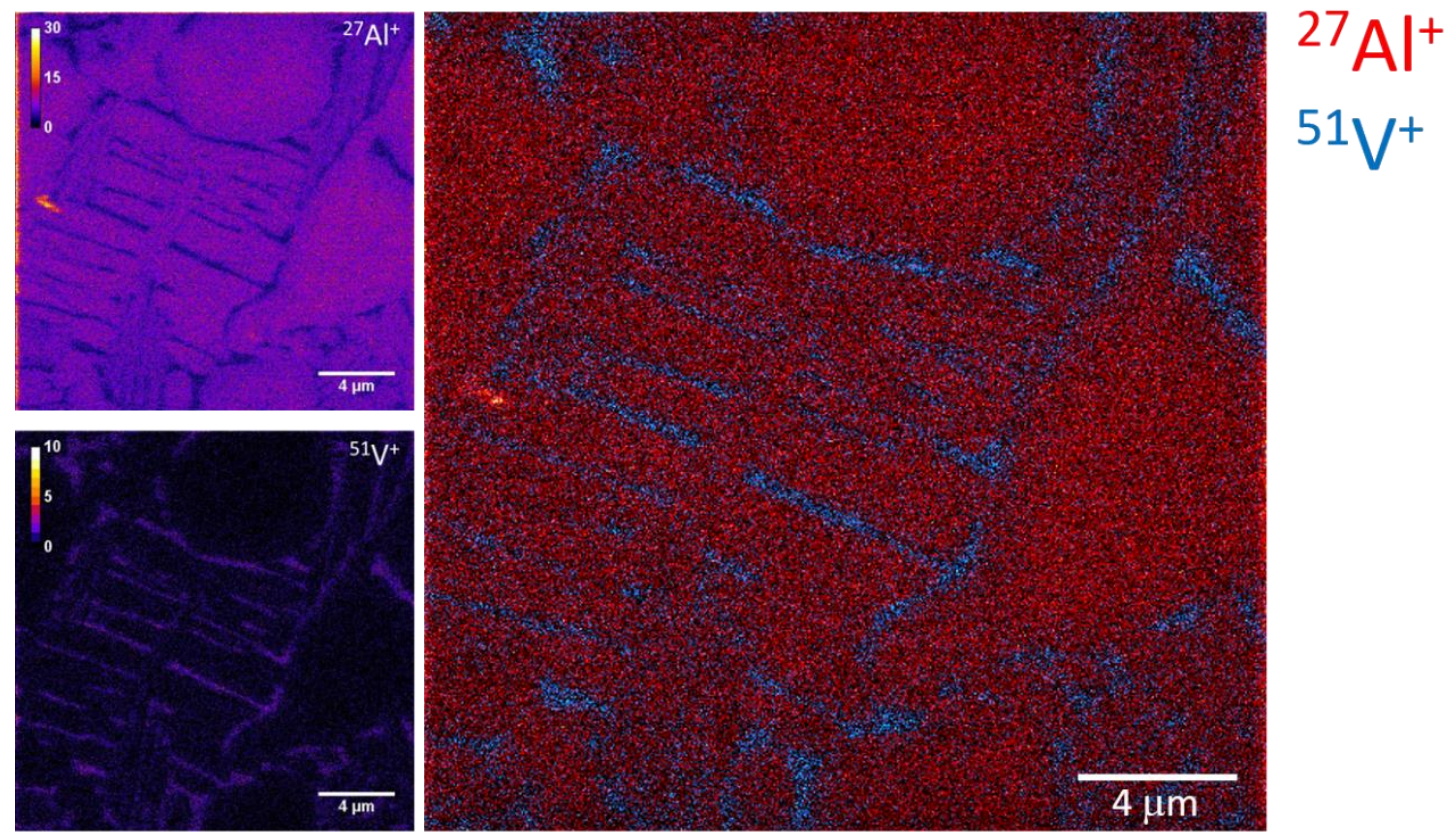

Figure 1. SIMS images of a dual-phase Ti-6Al-4V alloy to investigate phase transformation kinetics obtained on a DualBeam-SIMS (Thermo Fisher SCIOS with LIST magnetic sector SIMS). $16 \mathrm{keV} \mathrm{Ga+} \mathrm{beam,} 21 \mu \mathrm{m}$ field of view, 512x512 pixels, $2 \mathrm{~ms}$ dwell time per pixel. 

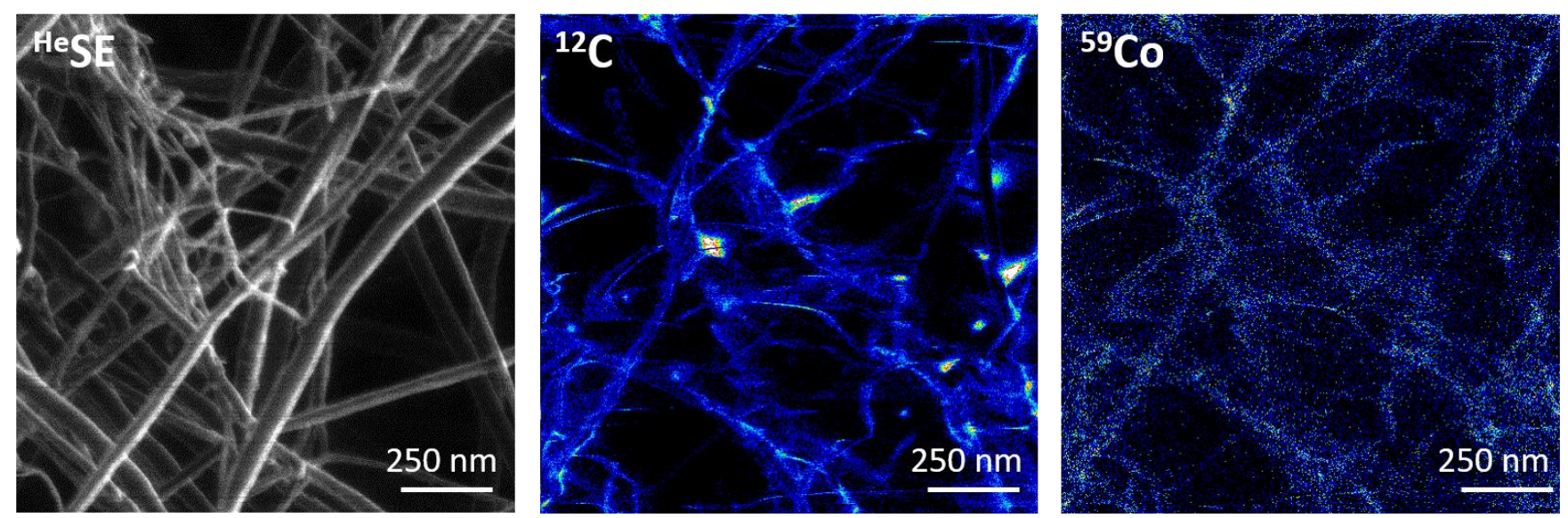

Figure 2. HIM-SIMS investigation of carbon nanotubes (CNT) selectively coated with cobalt nanocomposites: SE image obtained with a $\mathrm{He}$ + beam (30 keV, $0.2 \mathrm{pA}, 1024 \times 1024$ pixels), 12C and 59Co SIMS images obtained with a Ne+ beam ( $25 \mathrm{keV}, 1.5 \mathrm{pA}, 512 \times 512$ pixels). $2 \mu \mathrm{m}$ field of view.

\section{References}

[1] T. Wirtz, P. Philipp, J.-N. Audinot, D. Dowsett, S. Eswara, Nanotechnology 26 (2015) 434001

[2] F. Vollnhals, J.-N. Audinot, T. Wirtz, M. Mercier-Bonin, I. Fourquaux, B. Schroeppel, U. Kraushaar, V. Lev-Ram, M. H. Ellisman, S. Eswara, Anal. Chem. 89 (2017) 10702

[3] D. Dowsett, T. Wirtz, Anal. Chem. 89 (2017) 8957

[4] T. Wirtz, O. De Castro, J.-N. Audinot, P. Philipp, Ann. Rev. Anal. Chem. 12 (2019)

[5] E. Serralta, N. Klingner, O. De Castro, M. Mousley, S. Eswara, S. Duarte Pinto, T. Wirtz, G. Hlawacek, Beilstein J. Nanotechnol. 11 (2020) 1854-1864

[6] www.npscope.eu. This project has received funding from the European Union's Horizon 2020 Research and Innovation Programme under grant agreement No. 720964

[7] S. Eswara, A. Pshenova, L. Yedra, Q. H. Hoang, J. Lovric, P. Philipp, T. Wirtz, Appl. Phys. Revs. 6 (2019) 021312 